\title{
Endoscopic ultrasound-directed transgastric ERCP facilitating extensive ampullectomy in Roux-en-Y gastric bypass patient
}
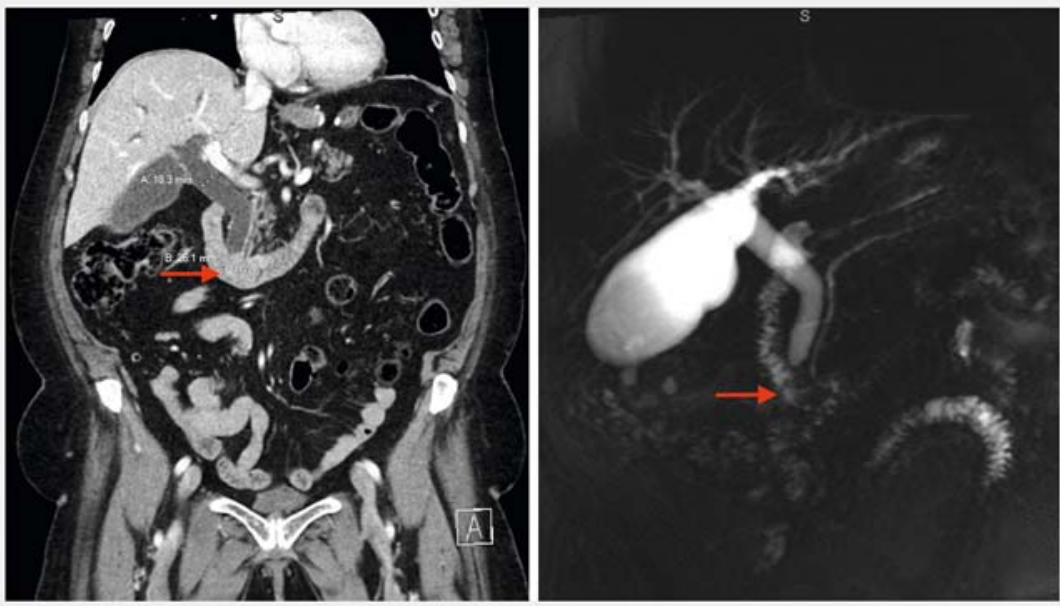

- Fig. 1 Computed tomography scan (left) showing a large ampullary mass (red arrow) that can be traced as a hypointense area on magnetic resonance cholangiopancreatography (right).
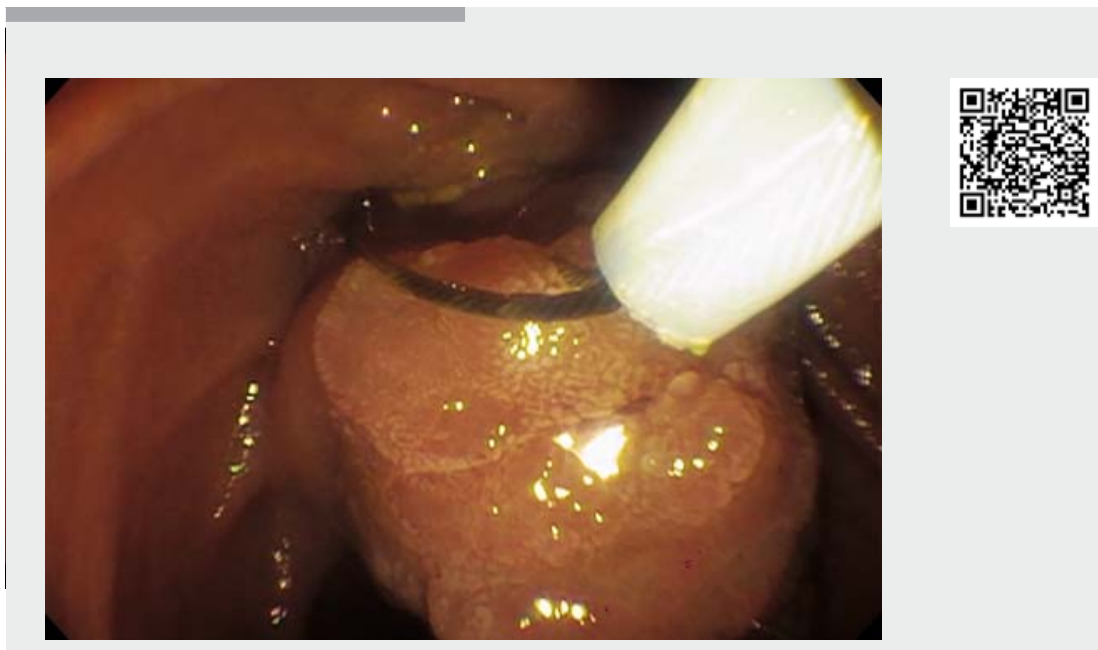

Video 1 Successful use of endoscopic ultrasound-guided gastrogastrostomy to secure ERCP access in gastric bypass anatomy. The extremely complex ampullectomy lasted more than 3 hours and required 2 advanced endoscopists.

A 71-year-old woman with a history of BRCA2 + ovarian cancer and Roux-en-Y gastric bypass was incidentally found to have transiently elevated cholestatic liver function tests. Magnetic resonance cholangiopancreatography (MRCP) demon- strated intra- and extrahepatic dilatation to the level of the pancreatic head but no apparent stone or mass. A contrast-enhanced abdominal computed tomography (CT) scan identified a 26-mm ampullary neoplasm (> Fig. 1). When re-

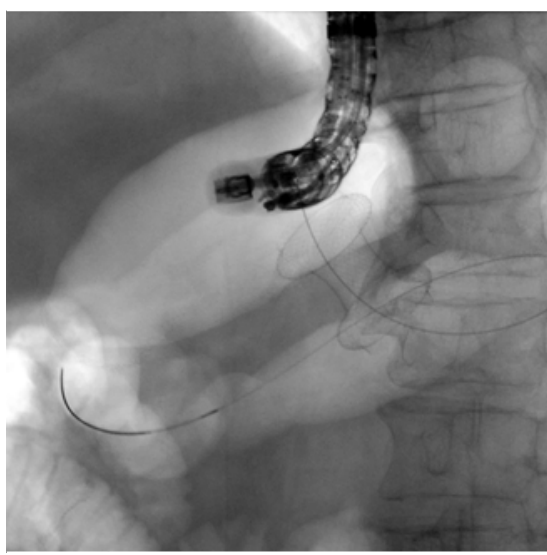

- Fig. 2 Fluoroscopic image demonstrates successful apposition of the gastric pouch and the remnant by a lumenapposing metal stent for the gastrogastrostomy.

viewed in retrospect, the lesion was present on her positron emission tomography (PET) scan 3 years ago with a mild increase in size.

BRCA2 mutation carriers are intrinsically at risk for malignancies, pancreatic among them [1]. Furthermore, ampullary lesions exceeding $15 \mathrm{~mm}$ carry a specificity of $80 \%$ for underlying malignancy [2]. Possible therapeutic strategies were reviewed considering various factors including altered anatomy. The Whipple procedure involved a risk for postoperative fistulae given her CT findings of fatty pancreas; she was also a poor surgical candidate. Percutaneous gastrostomy or laparoscopy-assisted endoscopic retrograde cholangiopancreatography (ERCP) would be technically challenging with ampullectomy intended. A multidisciplinary majority decision favored a complete endoscopic approach employing endoscopic ultrasound (EUS)-directed transgastric ERCP, typically a twostep technique including placement of a lumen-apposing metal stent (LAMS) reconnecting the stomach through temporary gastrogastrostomy with subsequent ERCP after tract maturation. If 

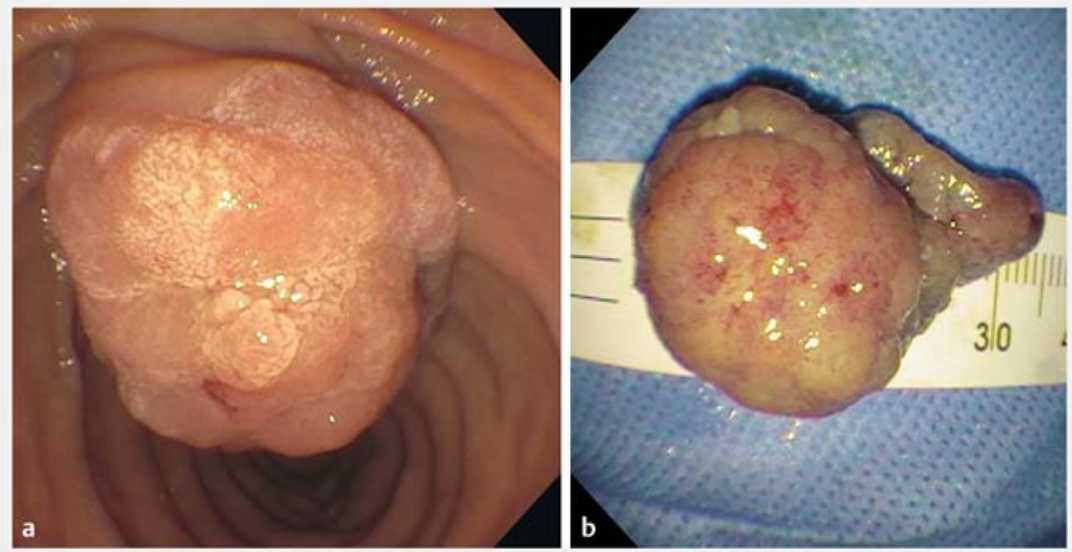

- Fig. 3 a Endoscopic view showing a 40-mm ampullary neoplasm with an adenomatous pattern. $\mathbf{b}$ A photo of the resected adenomatous tissue measuring $35 \mathrm{~mm}$.

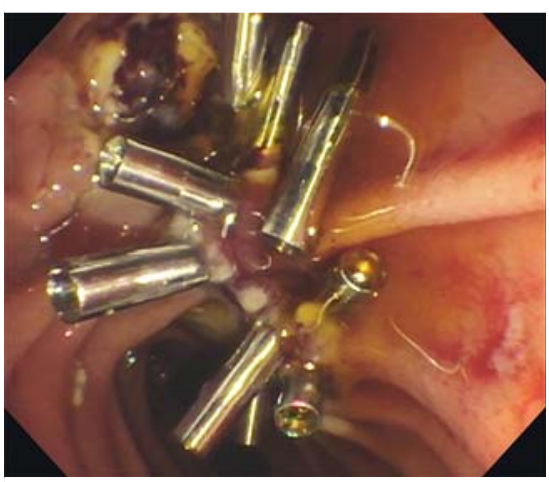

Fig. 4 Post-polypectomy defect closed in a zipper-like manner using hemostatic clips.

waiting is not an option, the procedure can be also done in a single session [3]. The technique carries a risk of weight gain by essentially reversing the bypass. However, supporting evidence is lacking, and any weight gain before fistula closure would likely be outweighed by the benefit of the procedure [4-5].

EUS-guided gastrogastrostomy was performed uneventfully ( $\triangleright$ Fig. 2) ( $\triangleright$ Video 1). After tract maturation, ERCP was performed with a near-complete resection of a 40-mm ampullary mass using hot snare polypectomy (\$Fig.3, \$ Fig.4). A small residuum remained and was removed in 4 weeks ( Fig.5). Histopathology showed ampullary adenoma nega-

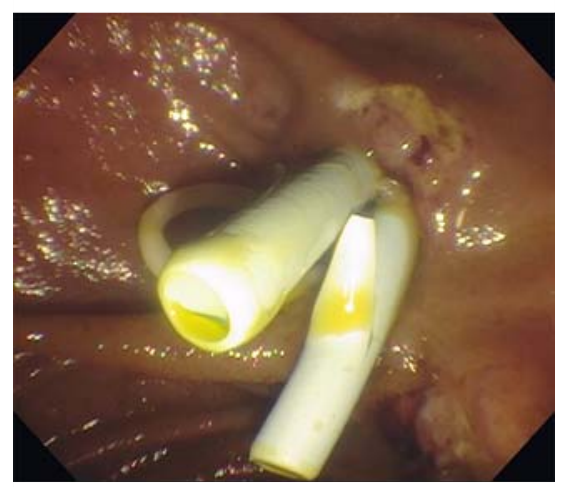

- Fig. 5 Endoscopic view showing 2 plastic biliary stents and a prophylactic pancreatic stent placed to secure adequate drainage after ampullectomy completion.

tive for high grade dysplasia or invasion, and the patient was scheduled for endoscopic follow-up with LAMS extraction and fistula closure in 2 months.

\section{Endoscopy_UCTN_Code_TTT_1AR_2AF}

\section{Competing interests}

M. L. Freeman is a consultant for Boston Scientific. The other authors declare they have no conflict of interest.
The authors

Petr Vaněk ${ }^{1,2}$, Martin L. Freeman ${ }^{1}$, Guru Trikudanathan ${ }^{1}$

1 Division of Gastroenterology, Hepatology and Nutrition, University of Minnesota, Minneapolis, MN, USA

2 2nd Department of Internal Medicine, Palacky University Olomouc and University Hospital Olomouc, Olomouc, Czech Republic

Corresponding author

\section{Guru Trikudanathan, MD}

Division of Gastroenterology, Hepatology and Nutrition, 420 Delaware Street SE, MMC 36, Minneapolis, MN 55455, USA Fax: +1-612-625-5620 triku001@umn.edu

\section{References}

[1] Iqbal J, Ragone A, Lubinski ] et al. The incidence of pancreatic cancer in BRCA1 and BRCA2 mutation carriers. Br J Cancer 2012; 107: 2005-2009

[2] $\mathrm{Kim} \mathrm{JH}, \mathrm{Kim} \mathrm{JH}$, Han JH et al. Is endoscopic papillectomy safe for ampullary adenomas with high-grade dysplasia? Ann Surg Oncol 2009; 16: 2547-2554

[3] Vanek P, Mallery S, Freeman ML et al. SingleSession Endoscopic Ultrasound-Directed Transgastric ERCP ("EDGE") in a Bariatric Patient with Pancreatic Mass and Biliary Obstruction. Obes Surg 2020. doi:10.1007/ s11695-020-04839-z

[4] Tyberg A, Nieto J, Salgado S et al. Endoscopic Ultrasound (EUS)-Directed Transgastric Endoscopic Retrograde Cholangiopancreatography or EUS: Mid-Term Analysis of an Emerging Procedure. Clin Endosc 2017; 50: 185-190

[5] Kedia P, Tarnasky PR, Nieto J et al. EUS-directed Transgastric ERCP (EDGE) Versus Laparoscopy-assisted ERCP (LA-ERCP) for Roux-en-Y Gastric Bypass (RYGB) Anatomy: A Multicenter Early Comparative Experience of Clinical Outcomes. J Clin Gastroenterol 2019; 53: 304-308

\section{Bibliography}

Endoscopy 2021; 53: E261-E262

DOI 10.1055/a-1252-2561

ISSN 0013-726X

published online 1.10 .2020

(C) 2020. Thieme. All rights reserved.

Georg Thieme Verlag KG, Rüdigerstraße 14,

70469 Stuttgart, Germany 